\title{
Selected current data on metronomic therapy (and its promise) from India
}

We refer to data on metronomic therapy from India and selected other abstracts mentioned below while discussing its current value and future promise.

\section{METRONOMIC THERAPY IN HEAD AND NECK CANCER}

An audit of the results of a triplet metronomic chemotherapy regimen incorporating a tyrosine kinase inhibitor in recurrent/metastatic head and neck cancers patients

\section{Geetha M, Satheesan Balasubramanian, Sajith Babu, Vinin N V, *Biji M S \\ Malabar Cancer Centre, *Email ID: vininnair@gmail.com}

BACKGROUND: Addition of erlotinib to metronomic chemotherapy (MCT) may lead to further improvement in progression free survival (PFS) and overall survival in head and neck cancers. The aim of this study was to study the PFS with MCT + erlotinib combination in our setting. METHODS: Single arm prospective observational study conducted at Malabar Cancer Center. Patients warranting palliative chemotherapy for head and neck cancers, having adequate organ function, not affording cetuximab and not willing for intravenous chemotherapy were included in this study. Oral methotrexate $\left(15 \mathrm{mg} / \mathrm{m}^{2} /\right.$ week $)$, oral celecoxib (200 mg twice daily) and erlotinib (I50 mg once daily) were administered till the progression of the disease or till intolerable side effects. Patients underwent toxicity (CTCAE version 4.02) and response (RECIST version I.I) assessment every 30 days. Statistical analysis was performed using SPSS version 16((IBM New York). Descriptive statistics and KaplanMeier analysis have been performed. RESULTS: A total of 15 patients received MCT.The median age of these patients was 65 years (range 48-80). The Eastern Cooperative Oncology Group performance status was $0-1$ in 7 patients (46.7\%), while it was 2 in 8 patients (53.3\%). The primary sites of tumor were predominantly oral cavity, II (73.4\%). Prior to MCT, treatment with palliative radiation therapy was given in II patients and curative treatment in 2 patients. The best response post MCT was complete remission in 2 patients, partial remission in 7 patients, stable disease in 4 patients, and progressive disease in 2 patients. The median estimated PFS was I 48 days ( $95 \%$ confidence interval $95.47-200.52$ days). For a median follow up of $18 \mathrm{I}$ days there were only three deaths. Grade 3-4 toxicity was seen in 6 patients (40\%). Dose reduction was required in 4 patients (26.7\%). CONCLUSION:The addition of erlotinib to a MCT schedule of methotrexate and celecoxib resulted in a promising PFS and should be tested in future studies

Outcomes of operable oral cavity cancer in rural India: A retrospective study

Pandey $A^{\prime}$, Desai $A^{2}$, Kulkarni $A^{2}$, Kulkarni $R^{2}$, Gargade $C^{2}$, Patil N², Ganoo $\mathbf{M}^{2}$, Ostwal V', *Banavali SD'

'Tata Memorial Hospital, Parel, Mumbai, ${ }^{2}$ BKL Walawalkar Hospital, Dervan, Chiplun, Ratnagiri, *Email ID: banavali_2000@yahoo.com

BACKGROUND: Oral cavity cancer is the most common cancer among rural India due to high tobacco consumption. There is paucity of data for outcomes of operable oral cavity cancer among rural population. AIM:To evaluate outcomes of operable oral cavity carcinoma. OBJECTIVES: To evaluate disease free survival (DFS) and factors affecting outcome in operable oral cavity cancer. MATERIALAND METHODS: Data of patients diagnosed with oral cavity cancer registered between May 2008 to May 2014 were retrieved from hospital based cancer database. Only those patients who presented with operable Oral cavity cancer and subsequent underwent upfront definitive surgery were included for analysis. Demographic profile, stage, tobacco consumption, adjuvant therapy and pattern of failure were collected. Kaplan Meir survival analysis was used to determine disease free survival and overall survival. Log rank test was used to evaluate factors affecting outcome. RESULTS: Median follow up is 24 months. Out of 335 patients, $225(67 \%)$ had advanced operable cancer with 42/225 (I8\%) and I83/225 (82\%) as stage III and IV respectively. Buccal mucosa was the most common sub-site (I78/335,53\%) followed by tongue $(63 / 335,19 \%) .92 \%$ patients were addicted to smokeless tobacco, while $27 \%$ were smokers and $27 \%$ were chronic alcoholics. Out of 225 patients who required adjuvant radiotherapy, 196(87\%) completed planned dose. Local relapse was most common (82/I27,65\%) followed by metastasis (19\%), regional (II\%) and loco regional failure (5\%). Median DFS is I 3 months with 2 yr relative DFS $32 \%$. Median Overall Survival is 30 months, with 2 yr OS of $54 \%$. Metronomic adjuvant oral chemotherapy was given in 130/225(58\%); Stage III and IVA patients with median of 12 months (3-18 months). Use of metronomic chemotherapy improved DFS( 8 vs 14 months, $p=0.22$ ) and overall survival. ( 14 vs 26 months, $\mathbf{p}=\mathbf{0 . 0 4}$ ) CONCLUSION: Oral cavity cancer is a major health care problem in rural India due to various smokeless tobacco preparations. Presentation at advanced stage leads to suboptimal outcomes. Benefit of metronomic maintenance chemotherapy in locally advanced oral cavity needs to be further evaluated prospectively.

Paclitaxel-Cetuximab versus oral metronomic chemotherapy as palliative chemotherapy in head and neck cancers - A match pair analysis.

\section{Vivek Agarwala,Vijay M Patil,Vanita Noronha,Amit Joshi,} Saurabh Zanwar, Vamshi Muddu, Sachin Dhumal, * Kumar Prabhash

Tata Memorial Hospital, *Email ID: kprabhash I@gmail.com

BACKGROUND: Cetuximab based palliative chemotherapy is the standard of care in metastatic head and neck cancers. However, only few patients can afford cetuximab in developing countries. A recent study showed the superiority of oral metronomic chemotherapywith daily celecoxib and weekly methotrexatein palliative setting over maximum tolerable dose chemotherapy which did not include cetuximab. The present match pair analysis was planned to compare the efficacy of cetuximab based chemotherapy versus metronomic therapy. METHOD: 60 patients with metastatic/recurrent head and neck squamous cell cancer treated with weekly Paclitaxel $(80 \mathrm{mg} / \mathrm{m} 2)$ and cetuximab were matched with 60 patients treated with oral metronomic chemotherapy.The matching was done on I:I basis for site of primary and event free period from prior treatment.The primary endpoint of the analysis was overall survival (OS). OS was estimated by Kaplan-Meier method. The cohorts were compared using the log-rank test.A multivariable Cox proportional regression model was used to identify independent factors affecting PFS and OS. RESULT:The median OS was I 9 I days (95\% Cl 122.2-259.8 days) in metronomic cohort and 256 days ( $95 \% \mathrm{Cl}$ I $77.0-334.9$ days) in cetuximab cohort. On cox proportional hazard model, ECOG PS (0-I versus 2 ) \& therapy (cetuximab versus metronomic) had a statistically significant impact on OS. The hazard ratio was 0.58 in favour of cetuximab cohort $(95 \% \mathrm{Cl} 0.35-0.95, p=0.031)$. CONCLUSION: Cetuximab based chemotherapy leads to significant improvement in OS as compared to metronomic chemotherapy in palliative chemotherapy of head and neck cancers.

\section{Metronomic chemotherapy in advanced/recurrent head and neck cancers - A tertiary centre experience}

\section{K.Kalaichelvi, S.Sureshkumar, G.Raja, K.S.Senthilkumar, *Kharthik $\mathbf{N}$ \\ Madras Medical College, Chennai, *Email ID: drnkharthik@gmail.com}

BACKGROUND: To assess the efficacy and toxicity of metronomic chemotherapy with oral methotrexate and celecoxib in patients with advanced / recurrent head and neck squamous cell carcinoma (HNSCC) MATERIALS AND METHODS: Patients who received metronomic chemotherapy from May 2014 to November 2015 for advanced/recurrent HNSCC were analysed retrospectively. The combination of weekly oral methotrexate $5 \mathrm{mg}$ twice daily for 2 days/week and oral celecoxib $200 \mathrm{mg}$ 
twice daily was offered as metronomic chemotherapy. The chemotherapy was either stopped on disease progression or intolerable side effects or unwillingness of the patient to continue. The efficacy was noted in terms of clinical benefit rate, pain control, changes in Quality of life, and median time to progression. The toxicity profile was graded with common terminology criteria for adverse events (CTCAE) version 4.03. EORTC Quality of life(QOL) questionnaires - QLQ -C30 and QLQ - H\&N35 were used to estimate the QOL scores. RESULTS: 30 patients ( 20 males, 10 females) were analysed.The median age was 58 years.At the end of four months, $60 \%$ had stable disease (SD), $10 \%$ had partial response(PR), and $30 \%$ had progressive disease(PD). The clinical benefit rate (PR+SD) was $70 \%$. The estimated median time to progression was eight weeks. The pain control was achieved in about $80 \%$ of patients. Only I patient had Grade III mucosal toxicity, II patients had Grade I/II mucosal toxicity and 18 patients had no mucosal toxicity. Mean QOL scores were improved with this metronomic chemotherapy. CONCLUSION: Oral metronomic chemotherapy with methotrexate and celecoxib for patients with advanced/recurrent HNSCC was effective, well tolerated, provides good pain control and improves Quality of life with least toxicity profile.

Nutrition and Oral Metronomic Chemotherapy in Advanced Head and Neck Cancer: Impact of Albumin and Hemoglobin Levels

\section{Sujith KM,Vijay Patil,Vanita Noronha,Amit Joshi, Sachin Dhumal, *Kumar Prabhash}

Tata Memorial Hospital, Mumbai, *Email ID: kprabhash I@gmail.com

BACKGROUND:To assess baseline nutritional status of advanced head and neck cancer patients and its implications to response after oral metronomic chemotherapy (OMCT). MATERIALS AND METHODS: A retrospective audit of 75 patients of advanced head and neck cancers who were treated with oral metronomic chemotherapy was conducted based on the electronic medical records. Patients who received at least 2 months of OMCT were included. RESULTS: The male to female ratio was 9: I. Median age was $48 \mathrm{yrs}$ (30-7lyrs). $65 \%$ of patients had oral cavity cancers. $40 \%$ had albumin $<4 \mathrm{~g} / \mathrm{dl}$. The mean baseline albumin was $4.02+1-0.6 .25 \%$ patients had $\mathrm{Hb}<1 \mathrm{Ig} / \mathrm{dl}$. The mean $\mathrm{Hb}$ was $12.4+/-1.27 .50 \%$ had $\mathrm{BMI}<18.5$ of which $21 \%$ had $\mathrm{BMI}<16$. Mean baseline BMI was $18.8+/-3.7$. Post 2 months of OMCT, symptomatic benefit was reported by $75 \%$ of patients and $25 \%$ had either no relief or had increase in symptoms. $30 \%$ of patients with albumin $<4 \mathrm{~g} / \mathrm{dl}$ had no symptomatic benefit with OMCT when compared to $20 \%$ of patients with albumin $>4 \mathrm{~g} / \mathrm{dl} .55 \%$ of patients with $\mathrm{Hb}<\mathrm{II} / \mathrm{dl}$ had no benefit with OMCT versus $20 \%$ of patients with $\mathrm{Hb}>\mathrm{I} \mathrm{Ig} / \mathrm{dl}$. Mean BMI of patients with albumin $<4 \mathrm{~g} / \mathrm{dl}$ was 16.6 which was significantly lower than patients with albumin $>4 \mathrm{~g} / \mathrm{dl}(20.2, \mathrm{p}=0.00 \mathrm{I})$. CONCLUSION: Half of patients with advanced head and neck cancer have co-existing malnutrition with one fifth severely malnourished. $40 \%$ of patients have low albumin levels. One fourth of patients have moderate anemia. Poor symptomatic response in patients with low baseline albumin and hemoglobin needs to be studied further to define them as prognostic factors. Early nutritional intervention even prior to start of OMCT or palliative chemotherapy is mandatory.

3 Drug Oral Metronomic Chemotherapy in Advanced Recurrent and Metastatic Head and Neck Squamous Cell Carcinoma

\section{*Shobana Sekhar, M. *Vamshi Krishna, Umanath K Nayak, Chinnababu Sunkavalli, Arsheed Hakeem, Kausik Bhattacharya, Nandita Sesikeran}

Apollo Cancer Institute, Hyderabad, *Email ID: shobanasekhar@gmail.com

INTRODUCTION: Patients with recurrent or metastatic HNSCC have poor prognosis. Standard cetuximab-based palliative chemotherapy is expensive and provides marginal benefit and significant toxicity. Studies using oral agents like erlotinib or methotrexate have shown promising results. We decided to study 3-drug oral combination in a metronomic schedule in patients unsuitable for cetuximab chemotherapy. OBJECTIVES: To determine progression free survival (PFS) and overall survival (OS) in patients with recurrent and metastatic HNSCC treated with 3-drug metronomic chemotherapy (MCT). MATERIAL AND METHODS: Patients were treated with MCT between October 2014 and March 2016.
Oral MCT included erlotinib I50 mg once daily, methotrexate I5-20 mg once a week and celecoxib $200 \mathrm{mg}$ twice daily and was continued until disease progression or intolerance. The primary endpoints were PFS and overall survival. Other endpoints were clinical response, tolerance and toxicity profile. RESULTS: I 3 patients with median age of 52 years (range 33-75), I I males and 2 females, received MCT.The primary site was tongue (5 patients), buccal mucosa (6), soft palate (I) and hypopharynx (I). The median time to relapse was 5 months (I-24 months). None of the patients received palliative chemotherapy. The reasons included nonaffordability, age and unwillingness for chemotherapy. The performance status was I in 8 patients and 2 in 5 patients. With a median follow up of 6 months, the estimated median PFS was 7.8 months and the median OS has not been reached. Twelve patients had clinical response after 2 months. Grade 3 rash was seen in 3 patients. Overall, dose reduction for erlotinib was done in 7 patients due to rash, mucositis or weakness. None of the patients needed hospitalization and were treated on outpatient basis. CONCLUSIONS: In patients with recurrent and metastatic HNSCC, 3 drug MCT as the initial therapy provided favourable clinical response and PFS with acceptable toxicity profile.

Weekly chemotherapy as Induction chemotherapy in locally advanced head and neck cancer for patients ineligible for 3 weekly maximum tolerable dose chemotherapy

Sanyo P Dsouza', Vijay Maruti Patil', Vanita Noronha', Amit Joshi',Vamshi Krishna Muddu', Sachin Dhumal', Supreeta Arya $^{2}$, Shashikant Juvekar ${ }^{2}$, P Pai ${ }^{3}$, Pankaj Chatturvedi ${ }^{3}$, Chaukar Devendra ${ }^{3}$,Sarbani Ghosh ${ }^{4}$, Anil D'cruz ${ }^{3}$, *Prabhash Kumar '

'Dept. of Medical Oncology, Tata Memorial Hospital, Mumbai, ${ }^{2}$ Dept. of Radio Diagnosis, Tata Memorial Hospital, Mumbai, ${ }^{3}$ Dept. of Head and Neck Surgical Oncology, Tata Memorial Hospital, Mumbai, ${ }^{4}$ Dept of Radiation Oncology, Tata Memorial Hospital, Mumbai,

*Email ID: kprabhashI@gmail.com

OBJECTIVE: To study the safety and efficacy of weekly chemotherapy as part of induction chemotherapy, in locally advanced head and neck cancerfor patients, who are unfit for upfront radical treatment. MATERIALS AND METHODS: It is a retrospective analysis of on-use weekly chemotherapyas Induction chemotherapy in locally advanced head and neck cancer, who are technically unresectable are unfit for upfront radical treatment.Induction chemotherapy given was a 2 drug combination of paclitaxel $(80 \mathrm{mg} / \mathrm{m} 2)$ and carboplatin AUC 2. The decision to give weekly inductionchemotherapy was given on the basis of presence of 2 more following features: Poor performance status (ECOG PS 2-3), presence of uncontrolled co morbidities, BMI below $18.5 \mathrm{~kg} / \mathrm{m} 2$ and age more than 60 years. The Statistical Package for the Social Sciences software (SPSS version 16.0) wasused for analysis. The response rates, toxicity(accordance with CTCAE vs. 4.02), completion rate $(C P)$ of radical intent treatment post neoadjuvantchemotherapy (NACT), progression-free survival (PFS) and overall survival (OS) are reported. RESULTS: Fifteen patients were considered for suchtherapy. Fourteen out of fifteen patients completed NACT. The median numbers of planned weekly cycles were 6 (3-8). Response (CR + PR) wasseen in 10 patients. Overall grade 3-4 toxicity was seen in 6 patients. No toxicity related mortality was noted. The calculated completion rate $(C p)$ of radical intent treatment post NACT was $46.7 \%$. The median PFS and OS were 10.36 months ( $95 \% \mathrm{Cl} 6.73-14.00$ months) and 16.53 months( $95 \% \mathrm{Cl} 4.22-28.84)$. CONCLUSION: Use of induction chemotherapy with weekly regimen is safe and effective in selected cohort of patients withlocally advanced disease who are unfit for upfront radical treatment.

Health-related quality of life in patients with metastatic, relapsed, or inoperable squamous cell carcinoma of the head and neck in India

Chandrakanth MV,Vanita Noronha,Amit Joshi , Shalaka Marfatia, Vijay Patil, Shashikant Juvekar, Supreeta Arya, Shripad Banavali, *Kumar Prabhash Tata Memorial Hospital, *Email ID: kprabhash I@gmail.com 
OBJECTIVE: The objective of this study was to evaluate changes in health related quality of life (HRQoL) in patients with metastatic head and neck $(\mathrm{H} \& N)$ cancer randomized to receive metronomic (methotrexate and celecoxib) or cisplatin chemotherapy. METHODS: Patients older than 18 years, with a Karnofsky Performance score of $\geq 70$, and diagnosed with metastatic, locally advanced inoperable or recurrent head and neck $(\mathrm{H} \& \mathrm{~N})$ cancer not amenable to surgery or radiation were randomized $(\mathrm{I}: \mathrm{I})$ to receive metronomic or cisplatin chemotherapy. All patients were recruited from the Tata Memorial Hospital, Mumbai, India. In addition to demographic and baseline clinical characteristics, patients were asked to rate their HRQoL using the EORTC QLQ-C30 and the EORTC QLQ-H\&N35 questionnaires (Indian versions) at baseline and at the end of each chemo cycle (every 3 weeks) till the end of study or early termination. RESULTS: Of the I 10 patients screened, 87 agreed to participate in the study. Mean age of the study population was 47.5 years (S.D. \pm I0.04) for the metronomic group and 47.2 years (S.D. \pm 9.89 ) for the cisplatin group. Overall quality of life was not significantly different between the two treatment groups from baseline to end of treatment. However, there was a statistically significant improvement in Pain QLQ-C30 score from baseline to week 3 $(O R=3.14, p=0.036)$ and week $6(O R=3.33, p=0.034)$ in the metronomic arm compared with the cisplatin arm. CONCLUSION: In addition to improvements in survival, understanding the impact of treatment options on changes in HRQoL is important as it can aid physicians in making treatment and rehabilitation decisions for patients with advanced inoperable $\mathrm{H} \& \mathrm{~N}$ cancer.

A prospective randomized phase II study comparing metronomic chemotherapy with chemotherapy (single agent cisplatin), in patients with metastatic, relapsed or inoperable squamous cell carcinoma of head and neck

\section{Noronha V', Patil VM', Joshi A', Muddu VK', Dhumal S', Bhosale B', Arya S', Juvekar S', Banavali S', D'Cruz A', *Prabhash K'.}

'Dept of Medical Oncology Tata Memorial Hospital, ${ }^{2}$ Dept of Radiology Tata Memorial Hospital, ${ }^{3}$ Dept of Surgical Oncology Tata memorial Hospital,

*Email ID: kprabhash I@gmail.com

BACKGROUND: Cetuximab based treatment is the recommended chemotherapy for head and neck squamous cell cancers in the palliative setting. However, due to financial constraints, intravenous (IV) chemotherapy without Cetuximab is commonly used in lesser developed countries. We believe that oral metronomic chemotherapy may be safer and more effective in this setting. METHODS:We conducted an open label, superiority, parallel design, randomized phase II trial comparing oral MCT [daily celecoxib $(200 \mathrm{mg}$ twice daily) and weekly methotrexate $(15 \mathrm{mg} / \mathrm{m} 2)]$ to intravenous single agent cisplatin (IP) $(75 \mathrm{mg} / \mathrm{m} 2)$ given 3 weekly. Eligible patients had head and neck cancers requiring palliative chemotherapy with ECOG PS 0-2 and adequate organ functions who could not afford Cetuximab. The primary end point was progression-free survival. RESULTS: I 10 Patients were recruited between July $201 \mathrm{I}$ to May 20I3, 57 randomized to the MCT arm and 53 to the IP arm. Patients in the MCT arm had significantly longer PFS (median I0I days, 95\% Cl: 58.2-I43.7 days) compared to the IP arm (median 66 days, $95 \% \mathrm{Cl}$; $55.8-76.1$ days) $(p=0.014)$. The overall survival $(O S)$ was also increased significantly in the MCT arm (median 249 days, $95 \%$ Cl: $222.5-275.5$ days) compared to the IP arm (median I52 days, $95 \% \mathrm{Cl}$ : 104.2-199.8 days) $(p=0.02)$. There were fewer grade $3 / 4$ adverse effects with MCT, which was not significant. (I8.9\% vs. $31.4 \%, P=0.14)$. CONCLUSION: Oral metronomic chemotherapy has significantly better PFS and OS than single agent platinum in the palliative setting.

\section{Metronomic Paclitaxel and Carboplatin for Adenoid Cystic Carcinoma}

\section{Siddharth Turkar,Vanita Noronha,Amit Joshi,Vijay Patil, *Kumar Prabhash}

Tata Memorial Hospital, *Email ID:- kprabhashI@gmail.com

INTRODUCTION: Adenoid cystic carcinoma (ACC) is a rare malignancy that arises from salivary glands of the head and neck region. Chemotherapy using one or a combination of drugs (cyclophosphamide, 5- fluorouracil, doxorubicin, mitomycin-C and cisplatin) has been used with some success and remission. We describe one patient treated with weekly paclitaxel and carboplatin for 10 months without progression with good tolerance and minimal toxicities. CASE REPORT:A 44-year-old male with no comorbidities diagnosed as adenoid cystic carcinoma of the right submandibular gland in June 2008. The patient underwent surgery followed by postoperative radiation 60 Gy/30 \#. The patient presented with bone metastasis at DI to D3 vertebra April 20I3- treated with palliative RT 20Gy/5\# in May 20I3. The patient then presented on July 2014 with bone pain, cough and dyspnoea. Patient was found to have lung, liver and vertebral metastasis. Patient was planned for palliative chemotherapy with weekly paclitaxel and carboplatin. After 4 weeks pain and cough decreased by $80 \%$. Post 10 weeks response CT scan was showing stable disease. Post 20 cycles response CT scan was suggestive of stable disease with good symptomatic control of disease. Patient progressed post 25 cycles of weekly chemotherapy clinically with increasing cough and bone pain as well as radiological in May 20I5.Patient had progression-free interval of 10 months with toxicities encountered during chemotherapy were grade I peripheral neuropathy, grade I nausea. CONCLUSION: The combination metronomic regimen of weekly paclitaxel and Carboplatin has promised and needs to be studied further.

\section{Metronomic palliative chemotherapy in maxillary sinus} tumor

Ashay Karpe, Vijay Patil,Amit Joshi, Vanita Noronha, Arun Chandrasekharan, Vikas Talreja, *Kumar Prabhash Tata Memorial Hospital, *Email ID: kprabhashI@gmail.com

BACKGROUND: Metronomic chemotherapy consisting of methotrexate and celecoxib recently has shown promising results in multiple studies in head and neck cancers. However these studies have not included patients with maxillary sinus primaries. Hence, the role of palliative a metronomic chemotherapy in patients with maxillary sinus carcinoma that is not amenable to radical therapy is unknown. METHOD :This was a retrospective analysis of carcinoma maxillary sinus patients who received palliative metronomic chemotherapy between August $201 \mathrm{I}$ and August 2014. The demographic details, symptomatology, previous treatment details, indication for palliative chemotherapy, response to therapy and overall survival details were extracted. SPSS version 16 was used for analysis. Descriptive statistics have been performed. Survival analysis was done by Kaplan Meier method. RESULTS: Five patients had received metronomic chemotherapy. The median age was 60 years (range 37-64 years). The proportion of patients surviving at 6 months, 12 months and 18 months were $40 \%, 40 \%$ and $20 \%$ respectively. The estimated median overall survival was 126 days ( $95 \% \mathrm{Cl} 0-299.9$ days). The estimated median survival in patients with an event free period after the last therapy of less than 6 months was 45 days while it was 409 days in patients with an event free period post last therapy above 6 months ( $p-0.063)$. CONCLUSION: Metronomic chemotherapy in carcinoma maxillary sinus holds promise. It has activity similar to that seen in head and neck cancers and needs to be evaluated further in a larger cohort of patients.

\section{METRONOMIC THERAPY IN OVARIAN CANCER}

Comparison of neo-adjuvant paclitaxel-platinum doublet versus oral metronomic therapy in advanced epithelial ovarian carcinoma: Rural cancer centre experience

\section{Pandey A', Jandyal $S^{1}$, Desai A², Kulkarni A², Kulkarni R², Gargade $C^{2}$, Patil N' ${ }^{2}$, Ganoo $\mathbf{M}^{2}$, Ostwal ' ', *Banavali SD'}

'Tata Memorial Hospital, Parel, Mumbai, ${ }^{2}$ BKL Walawalkar Hospital, Dervan, Chiplun, Ratnagiri, *Email ID: banavali_2000@yahoo.com

BACKGROUND AND RATIONALE: Neo-adjuvant paclitaxel- platinum doublet is the standard accepted therapy for advanced epithelial ovarian carcinoma. Due to poor socioeconomic profile and therapeutic constraints in rural centre, alternative low cost oral chemotherapy has been explored in such patients. AIM: To compare outcomes with neo-adjuvant paclitaxel -platinum doublet versus oral metronomic therapy. OBJECTIVES: To calculate Progression free survival (PFS) and factors affecting outcomes with comparison between neo- adjuvant chemotherapy versus oral metronomic therapy. MATERIAL AND METHODS: Data of patients diagnosed as epithelial ovarian carcinoma and registered between March 2009 and March 2014 were retrieved from hospital based cancer database. Demographic profile, stage, histology, chemotherapy types, response to chemotherapy, type of surgery and disease progression details was collected. Only patients who 
completed at least three cycles of chemotherapy with response evaluation and underwent surgery were selected for final analysis. Kaplan Meir survival analysis was used to determine progression free and overall survival. Log rank test was used to evaluate factors affecting outcome. RESULTS: Median follow up is 20 months. $26 / 56$ patients $(47 \%)$ received oral metronomic therapy while $30 / 56$ patients (53\%) received paclitaxel-platinum doublet as neo-adjuvant therapy. Chemotherapy in 5/26(19\%) patients had to change to paclitaxel-platinum from oral metronomic due to suboptimal response before cytoreductive surgery. 8/2I patients with exclusive oral metronomic neo-adjuvant therapy went on to receive metronomic therapy(MM) as adjuvant while remaining patients $(\mid 3 / 2 I)$ received six cycles of paclitaxel-platinum as adjuvant therapy(MC). Overall best response (GCIG criteria) with neo-adjuvant metronomic therapy are; 5/2 I CR, I4/2I PR and 2/2I SD, while the same with paclitaxel-platinum doublet were; 5/35 CR and 29/35 PR respectively. Median Progression free survival (PFS) with neo-adjuvant paclitaxel-platinum versus oral metronomic therapy was 19 and 13 months respectively $(p=0.38)$. PFS of patients who received adjuvant paclitaxel-platinum after oral metronomic therapy showed trend to better outcome compared to those who received metronomic therapy in both settings. ( 16 months versus 13 months, $p=0.87$ ) CONCLUSION: Oral metronomic therapy as neo-adjuvant therapy is a feasible option with acceptable outcomes in resource constraints setting. Studies are required to further explore outcomes with metronomic therapy compared to standard induction chemotherapy.

\section{A Phase II study of oral metronomic combination therapy in relapsed epithelial ovarian cancer}

\section{*Sudeep Gupta, Gautam Goyal, Jaya Ghosh, Jyoti Bajpai, Amita Maheshwari, Rajendra Kerkar, Kedar Deodhar, Bharat Rekhi, Santosh Menon, Shripad Banavali \\ Tata Memorial Centre, Mumbai.*Email ID: sudeepgupta04@yahoo.com}

BACKGROUND: Etoposide (E), cyclophosphamide (C) and tamoxifen (T) have been used as single agents in relapsed epithelial ovarian cancer (EOC) patients. We tested a low dose, continuous, daily, oral metronomic regimen that combined these 3 agents. METHODS: This single centre prospective study included patients with relapsed EOC with exposure to at least 2 prior lines of chemotherapy (CT) and at least partial as the best response to most recent regimen. Study regimen comprised daily oral administration of $E(50 \mathrm{mg} / \mathrm{m} 2)$ and $\mathrm{C}(50 \mathrm{mg} / \mathrm{m} 2)$ for 21 days of a 28 day cycle plus $T(20 \mathrm{mg} / \mathrm{m} 2$, twice daily) continuously. Primary endpoint was serological (CA-125) progression-free survival (PFS) as per Rustin Criteria and secondary endpoints were radiological (RECIST) PFS, overall survival (OS), duration of response (DOR), response rates and toxicity. RESULTS: Twenty six patients with a median age of 48 years were accrued, of whom 21 had received 2 prior lines of CT and 5 had received 3 lines. 25 evaluable patients received a median of 7 (I-20) cycles of metronomic regimen. The median delivered relative dose intensities of $\mathrm{E}, \mathrm{C}$ and $\mathrm{T}$ were $0.63,0.67$ and 0.92 respectively. Thirteen (52\%) patients needed dose reduction after a median of $3(\mathrm{I}-9)$ cycles. Common grade 3 or 4 toxicities included anemia, neutropenia, febrile neutropenia, nausea and diarrhoea in $44 \%, 40 \%, 16 \%, 16 \%$ and $12 \%$ patients respectively. Nineteen (76\%) patients had serological CR or PR with a median time to response (TTR) and DOR of I.8 $(95 \% \mathrm{Cl}, 0.83-2.96)$ and $7.0(95 \% \mathrm{Cl}, 6.0-8.0)$ months respectively. Eleven (46\%) of the 24 evaluable patients achieved radiological CR or PR with median TTR and DOR of $3.7(95 \% \mathrm{Cl}, \mathrm{I} .8-7.2)$ and $8(95 \% \mathrm{Cl}$, 4.8-I I.I) months respectively.Twenty four, 24 and 22 patients have respectively experienced serological progression, radiological progression and death at the time of analysis. The median serological PFS, radiological PFS and OS are 8.6 (95\% Cl, 6.6-10.7), 8.I (95\% Cl, $5.4-10.9)$ and 19 (95\% Cl, I 3.6-24.4) months, respectively. CONCLUSIONS: The oral metronomic combination of E, C and $\mathrm{T}$ has substantial and durable activity in relapsed $\mathrm{EOC}$ and is worthy of further evaluation.

\section{Metronomic oral cyclophosphamide (MOC) in recurrent and advanced ovarian cancer patients: A retrospective study}

Kalaichelvi K, Lakshminarasimman N, Ramkumar, Sureshkumar S, Raja G, *Arun Ramanan V

Madras Medical College and Institute Of Gynecology, Chennai, *Email ID: arun.ramanan8I@gmail.com
BACKGROUND:To study the efficacy of oral cyclophosphamide in recurrent and advanced ovarian cancer patients. MATERIALAND METHODS: Recurrent and advanced ovarian cancer patients who are unfit for intensive chemotherapy were prescribed oral cyclophosphamide at the dose of $50 \mathrm{mg}$ twice daily for 10 days, cycled every 28 days.Treatment-related toxicity assessed by $\mathrm{NCl}-\mathrm{CTC}$ criteria and response to treatment were assessed by RECIST criteria and subjective assessment. Progression-free (PFS), and overall survival (OS) were assessed. RESULTS: 7I patients (jan 2012 to mar 20I5) with mean age of $61.2 \pm 0.74$ years were analyzed: 44 patients $(61.9 \%)$ were platinum refractory/ resistant, while 26 patients $(36.6 \%)$ were platinum sensitive; 60 patients (84\%) had received atleast I previous chemotherapy before starting MOC. The objective response rate (ORR) (stable disease and partial response) was 20.4\%. 22 patients (30.9\%) experienced stable disease and 17 had response duration $\geq 6$ months, 8 patients had continued response for more than I year. 49 patients (69.01.\%) progressed during treatment. Median PFS was 5 months (range 2 - 25 months), and the 12-month PFS rate was I I\%; Median OS was 7 months, and the 12-month OS rate was $26 \%$ Median PFS for patients who responded to MOC was 9 months where as it was only 2 months for those who did not $(p=0.01)$. Median OS was 14 months for responding patients whereas it was 8 months for patients progressed on MOC $(p=0.02)$. No significant toxicity was observed. CONCLUSIONS: MOC is an effective therapy in the palliative treatment of recurrent and advanced ovarian cancer patients, who are unfit for intensive chemotherapy.

Oral metronomic cyclophosphamide in heavily treated recurrent carcinoma ovary: An old drug with a new concept

\section{Shobana Sekhar, M.Vamshi Krishna, Umanath K Nayak, Chinnababu Sunkavalli, Arsheed Hakeem, Kausik Bhattacharya, Nandita Sesikeran, *Talele Avinash Dnyandeo, *Rahul S Kulkarni, *Pushpak Chirmade}

Apollo Cancer Institute, Hyderabad *Email ID:- drguddu I@yahoo.com

INTRODUCTION: Patients with recurrent or metastatic HNSCC have poor prognosis. Standard cetuximab-based palliative chemotherapy is expensive and provides marginal benefit and significant toxicity. Studies using oral agents like erlotinib or methotrexate have shown promising results. We decided to study 3-drug oral combination in a metronomic schedule in patients unsuitable for cetuximab chemotherapy. OBJECTIVES: To determine progression free survival (PFS) and overall survival (OS) in patients with recurrent and metastatic HNSCC treated with 3-drug metronomic chemotherapy (MCT). MATERIAL AND METHODS: Patients were treated with MCT between October 2014 and March 2016. Oral MCT included erlotinib $150 \mathrm{mg}$ once daily, methotrexate I5-20 mg once a week and celecoxib $200 \mathrm{mg}$ twice daily and was continued until disease progression or intolerance. The primary endpoints were PFS and overall survival. Other endpoints were clinical response, tolerance and toxicity profile. RESULTS: I 3 patients with median age of 52 years (range 33-75), II males and 2 females, received MCT. The primary site was tongue (5 patients), buccal mucosa (6), soft palate (I) and hypopharynx (I). The median time to relapse was 5 months (I-24 months). None of the patients received palliative chemotherapy. The reasons included nonaffordability, age and unwillingness for chemotherapy. The performance status was $I$ in 8 patients and 2 in 5 patients. With a median follow up of 6 months, the estimated median PFS was 7.8 months and the median OS has not been reached. Twelve patients had clinical response after 2 months. Grade 3 rash was seen in 3 patients. Overall, dose reduction for erlotinib was done in 7 patients due to rash, mucositis or weakness. None of the patients needed hospitalization and were treated on outpatient basis. CONCLUSIONS: In patients with recurrent and metastatic HNSCC, 3 drug MCT as the initial therapy provided favourable clinical response and PFS with acceptable toxicity profile.

\section{METRONOMIC THERAPY IN BREAST CANCER}

Outcomes of breast carcinoma in rural India: A retrospective study

Pandey A', Desai A², Kulkarni A², Kulkarni $\mathbf{R}^{2}$, Gargade $\mathbf{C}^{2}$, Patil N², Ganoo $\mathbf{M}^{2}$, Ostwal V', *Banavali SD' 
'Tata Memorial Hospital, Parel, Mumbai, ${ }^{2}$ BKL Walawalkar Hospital, Dervan, Chiplun, Ratnagiri, *Email ID: banavali_2000@yahoo.com

BACKGROUND: Breast cancer is the second most common cause of cancer among rural women. There is lack of data about outcomes of breast cancer in rural India. AIM:To evaluate outcomes of breast cancer. OBJECTIVE:To evaluate progression free survival, overall survival and factors affecting outcome. MATERIAL AND METHODS: Data of patient diagnosed with invasive breast carcinoma and registered between March 2008 and May 2014 were retrieved from hospital based cancer database. Demographic profile, stage, classification, hormonal profile, chemotherapy, radiotherapy, type of surgery and disease progression details were collected. Kaplan Meir survival analysis was used to determine progression free survival and overall survival. Log rank test was used to evaluate factors affecting outcome. RESULT: Median follows up is 40 months. Out of 208 patients, 90 (43\%) were Operable breast cancer, while $98(47 \%)$ and 20 (10\%) had locally advanced and Metastatic breast carcinoma. Luminal A subtype was most common (92/193,48\%), followed by TNBC $(59 / 193,30 \%)$ and her2neu type $(30 / 193,16 \%)$. Majority of patients underwent MRM (I86/I89, 98\%) with only 3 patients had BCT done. $99 / / 88$ patients (53\%) required neo adjuvant chemotherapy, CAF $(98 \%)$ before definitive surgery with complete pathological response in $10 \%$. Adjuvant chemotherapy was delivered in 178/188(95\%) patients. 123/I47 (84\%) patients completed planned radiotherapy. Median progression free survival and overall survival are 55 and 75 months respectively with 5 year PFS and OS of $28 \%$ and $58 \%$ respectively. Luminal A subtype had best OS, with Her2neu type the worst (75 vs 27 months, $p=0.01$ ). Use of metronomic chemotherapy in TNBC (33/55 patients) improved overall survival (PFS, not reached Vs 29 months, $p=0.02$ ). CONCLUSION: Outcome of breast cancer in rural India is inferior compared to urban population due to advanced stage at diagnosis and lack of awareness. Use of metronomic chemotherapy in TNBC has potential to improve outcome which require further exploration.

\section{Oral Metronomic therapy in metastatic triple negative breast cancer (TNBC)}

\section{*Itesh Khatwani, Harsha Panchal,Asha Anand, Sonia Parikh, Avinash Talele, Kshitij Joshi, Preetam Kalaskar, Sandeep Kakkad,Alpesh Kikani, Gautam Makadia, Ekta Vala \\ Gujarat Cancer And Research Institute, Ahmedabad, \\ *Email ID: khatwaniitesh@gmail.com}

BACKGROUND: The therapeutic concept of administering agents (cytotoxic/-static, non-cytotoxic and/or targeted drugs) continuously at lower doses - relative to Maximum Tolerated Doses(MTD) without drugfree breaks over extended periods - known as 'metronomic therapy' (MT). Oral MT is particularly attractive due to its ease of administration and low toxic burden. Its main mechanism of action is antiangiogenesis rather than a classical antiproliferative effect like standard MTD-based regimens. PATIENTS AND METHODS: A retrospective analysis of appropriately selected 52 patients with metastatic triple negative breast carcinoma was carried out with the aim of reporting activity in terms of response rate, control of tumor-related symptoms, outcome, and toxicity.All patients had metastatic breast disease and had previously received at least one line of chemotherapy. All patients had received oral cyclophosphamide at $50 \mathrm{mg}$ daily plus oral low-dose methotrexate at $12.5 \mathrm{mg} / \mathrm{m} 2$ orally once a week. RESULTS: Overall response(PR+CR) according to the RECIST criteria I.I was seen in $25 \%$ of patients with a median duration of 7 months(range $5-14$ months), stable disease was seen in $38 \%$ of cases with a median duration of 9 months(range 5-14 months); thus accounting for a clinical benefit rate of $63 \%$. Grade $3 / 4$ hematological toxicity was most common and included non-febrile neutropenia in $5(10 \%)$ cases, anemia and thrombocytopenia in $2(3 \%)$ cases; all being easily manageable. One $(2 \%)$ patient developed MTX induced hypersensitivity pneumonitis. CONCLUSION: Although retrospectively recorded, this study supports the use of oral metronomic chemotherapy for patients with metastatic breast cancer. Significant clinical response was seen even in heavily pretreated patients with easily manageable grade 3-4 side-effects. Further studies are warranted to optimize the treatment schedule and to select patients who may benefit from such an approach.

\section{METRONOMIC THERAPY IN LUNG CANCER}

\section{A Pilot Study to Evaluate Efficacy and Safety of Low Dose Cyclophosphamide as Maintenance Treatment in Patients with Advanced NSCLC}

Talele Avinash D, Asha S Anand, Harsha P Panchal, Apurva A Patel, Sandeep Shah, Sonia K Parikh, Rahul S Kulkarni, Sandeep Kukkar, Mukesh J Choudhary, Pritam B Kalaskar, Alpesh Kikani, Mangesh M Mekha, Kshitij C Joshi, Bhushan Bharatri, Itesh Khat, *Prabhat Singh Malik

Gujarat Cancer and research institute, Regional cancer centre, B J Medical College, Ahmedabad, *Email ID:- drprabhatsm@gmail.com

INTRODUCTION: Metronomic therapy comprises of continuous administration of low dose chemotherapy in oral form has recently gained attention, due to its modest activity, convenience, use in outpatient setting and fewer hospital visits while freedom from iv infusion, minimum adverse effects are an added privilege. Metronomic therapy exerts its antitumour activity mainly due to it antiangiogenic effects, antitumour immunity and apoptosis; hence it is also described as mutitargetd therapy. OBJECTIVES: To evaluate safety and efficacy of oral metronomic cyclophosphamide in heavily treated recurrent ovarian cancer. Primary objective was to assess the progresson free survival and objective response rate $O R R(C R+P R)$, and clinical benefit rate $C B R(C R+P R+S D)$. Secondary objective was adverse events of therapy and compliance. MATERIAL AND METHODS: Retrospective analysis of patients receiving oral Cyclophosphamide $50 \mathrm{mg}$ /day. The therapy was continued till disease progression or unacceptable toxicity; whichever was earlier. The data was collected from Jan 2014 to Oct 20I5. Only patients previously treated with one or more platinum, Paclitaxel drug regimen and having evidence of recurrence were included.All patients had ECOG Performance status $<2$, adequate bone marrow reserve, ANC $>1500$, platelet count $>$ Ilakh and bilirubin, liver enzymes and creatinine $<1.5$ times of their upper normal limits. Response evaluation was done at 3 months or as advised by the treating physician if indicated early. Response to treatment was assessed by RECISTI.0 criteria and toxicity was graded according to CTCAE version 4.0. PFS was calculated from date of starting the therapy till the first documentation of disease progression. RESULTS: Total 24 patients were studied out of which 15 (62\%) were Platin sensitive (unwilling for parenteral CT) and $9(37.5 \%)$ were platin refractory. Median age of cohort was 52 years and 5, 16 and 3 patients had received 2,3 and 4 lines of therapy respectively.About $66 \%$ of patients were pre-treated with 3 lines of therapy. ORR of total patients was $4 / 24(16 \%)$ with CBR of $8 / 24(37 \%)$. Totally, I 2/24(50\%) had PD, 5/24(20\%) SD, 3/24(I2\%) PR and I/24(4\%) had CR. Median PFS was equal, 5 months in platinum sensitive and resistant sub group, and I0/24(4I\%) had PFS of $>6$ mths.Among Platin sensitive, $6 / 15(40 \%)$ patients had PD and 4/I5(26\%), 2/I5(I3\%), I/I5(6\%) had SD , PR, CR, with ORR, CBR of $3 / 15(20 \%)$ and $6 / 15(40 \%)$ respectively. In Platin refractory, 6/9(66\%) had PD and I/9(I I\%), I/9(II\%), 0 had SD, PR, CR, with ORR, CBR of $8 / 9(22 \%)$ respectively. All patients tolerated therapy well while I patient developed grade III neutropenia and I had grade II hepatic dysfunction. None of the patients had grade IV toxicity. CONCLUSIONS: Metronomic oral Cyclophosphamide is well tolerated and has modest efficacy in heavily pretreated ovarian cancer. Similar PFS in Platin sensitive and Platin refractory disease could be due to less number of Platin refractory patients. None the less it seems a promising option warranting further studies.

Efficacy and safety of metronomic administration of paclitaxel for advanced recurrent non-small-cell lung cancer

\section{Saurabh Zanwar, Vanita Noronha,Vijay Patil,Amit Joshi, Nilendu Purandare, *Kumar Prabhash}

Tata Memorial Centre, Mumbai, *Email ID: kprabhash I@gmail.com

CONTEXT: There are limited effective therapeutic options in the relapsed setting for non-small cell lung cancer (NSCLC) or in the first line for platinumineligible patients. AIM:To evaluate the safety and efficacy of a metronomic schedule of paclitaxel administered weekly in relapsed refractory NSCLC 
or upfront in patients not eligible for platinum-based chemotherapy. Settings and Design: Retrospective analysis of a prospectively collected database from the medical oncology department at Tata Memorial Hospital in Mumbai, India. MATERIALS AND METHODS: Patients with recurrent and treatmentnaïe platinum-ineligible advanced NSCLC were treated with weekly paclitaxel at $80 \mathrm{mg} / \mathrm{m} 2$ with palliative intent. Restaging scans were obtained every two months. Chemotherapy was continued until progressive disease, intolerable side effects, or decision of the patient. STATISTICALANALYSIS USED: SPSS version 16 was used for analysis. Simple percentages were used for descriptive statistics. Progression-free survival (PFS) was calculated from date of start of paclitaxel till the date of progression, change of therapy due to any reason, or death due to any cause. Overall survival (OS) was calculated from date of start of paclitaxel to death. The Kaplan Meier method was used for estimation of survival. RESULTS: There were 37 patients over eight months. The median age was 59 years, with a male-to-female ratio of 5:I.Two patients received paclitaxel in the first line, 18 patients in second line, nine in third line, five in fourth line, and three were in fifth line. $73 \%$ patients had received prior platinum and $48.6 \%$ patients had Eastern Cooperative Oncology Group performance status (ECOG PS) $>2$. The median number of weekly cycles delivered was 14 . The response rate was 35\% [complete remission (CR): $2.7 \%$, partial remission (PR): $32.4 \%$, stable disease (SD): $32.4 \%$, progressive disease (PD): $27 \%$, the median PFS was four months, and the estimated median OS was seven months. Chemotherapy was well tolerated. The most frequent grade 3 toxicities included anemia: $8 \%$, neutropenia: $5.4 \%$, and sensory neuropathy: $8 \%$. There were no grade 4 toxicities and no episodes of febrile neutropenia. CONCLUSIONS:Weekly low-dose continuous metronomictype scheduling of paclitaxel is safe and effective for relapsed refractory NSCLC and in the first line in platinum-ineligible patients.

Weekly Paclitaxel as a Second Line Agent in Relapsed Small Cell Lung Cancer - A retrospective tertiary care centre experience

\section{Arvind Sahu, Vijay Patil, Amit Joshi, Vanita Noronha, Sunny Jandyal, Nikhil Pande, *Kumar Prabhash \\ Tata Memorial Hospital, *Email ID: kprabhashI@gmail.com}

BACKGROUND: Topotecan has been standard of care as a second line agent in relapsed small cell carcinoma. But the availability and the cost of the drug has been a prohibitive factor for the use of this drug in the second line setting. Paclitaxel has shown antitumor activity in heavily pre-treated patients with SCLC. Phase II study of weekly paclitaxel in pre-treated SCLC patients has shown a median OS of 5.8 months and was very well tolerated. MATERIAL AND METHODS: Fifty seven patients of relapsed small cell cancer patients who presented to the Thoracic Medical Oncology Unit of Tata Memorial Centre, Mumbai with weekly paclitaxel at a dose of $80 \mathrm{mg} /$ $\mathrm{m} 2$ during the period of January,20 I I to December,20 I5. The patients were evaluated for baseline demographic data, previous treatment history, response rate, progression free survival, overall survival and toxicity to weekly paclitaxel. The data was collected from a prospectively maintained database in the Thoracic Medical Oncology Unit and was analysed using SPSS Version 16. RESULTS: Median age of the cohort was 58 years ( $40-77$ years). Etoposide carboplatin was the regimen used in 40patients $(70.2 \%)$ while the rest 17 patients had received Etoposide - Cisplatin (29.8\%). ECOG PS at relapse was $0 \mathrm{l}$ in $3(5.3 \%), 2$ in 49 (86.0\%) and 3 in 5 (8.7\%) patients. The response rate and clinical benefit rate were $8.8 \%$ (5 patients) and 50.9\% (9 patients) respectively. Gr $3 / 4$ toxicities were seen in $10.5 \%$ (6 patients) of patients. The median PFS was 145 days $(95 \% \mathrm{Cl}$ I I 6.6 - I 73.5 days) while the median OS was 168 days (95\% Cl II 2.5-223.5 days). CONCLUSION: Weekly Paclitaxel as a second line agent in relapsed small cell cancer of lung was a feasible and well tolerated agent.

\section{METRONOMIC THERAPY IN LYMPHOMA AND LUEKEMIA}

\section{Temozolamide based metronomic therapy for relapsed Hodgkin Lymphoma}

\section{*Prakash Chitalkar, Deeapak Singla, Prashant Kumbhaj, Novak Gupta, Renu Mishra G Voshesh}

Sri Aurobindo Medical College and PG Institute Indore (MP),

*Email ID:- prakashchitalkar@gmail.com
BACKGROUND: The pharmacological homology of temozolamide with Dacarbazine and its property to overcome the blood brain -barrier, are characteristics worth harnessing in patients with Hodgkin Lymphoma who have relapsed after multiple lines of therapy or after an Autologous stem cell transplant. PATIENTS AND METHODS: Since 2002, over thirteen years we have treated seven patients with relapsed Hodgkin Lymphoma, with metronomic therapy consisting of temozolamide $100 \mathrm{mg}$ per day for $7 / 28$ days; Cyclophosphamide $50 \mathrm{mg}$ twice a day for 21 / 28 days; and etoposide $50 \mathrm{mg}$ on alternate for 10 doses (20/28 days )Three males and four females; age median II (6to 19 years). Two patients entered after relapse following Autologous stem cell transplant. All patients completed at least six cycles; one patient completed nine cycles; Responses were documented with PET/ CT in four and CT scan in the remaining three. RESULTS: Two complete responses were documented. Five patients had partial responses The CRs lasted four months in the first patient, but is continued for 67 months in the other patient ( 27 year old female now) The remaining patients progressed and died after a median of II months (range 4-23 months) The long term survivor is alive and free ogf disease, with ovarian failure, and severe osteoporosis, though is active an $d$ retains a job. CONCLUSIONS: Metronomic therapy based on temozolamide is active in relapsed Hodgkin Lymphoma

\section{Theranostic approach for relapsed APDS like Burkitt lymphoma}

\section{Zapletalová D, *Melichárková K, Štěrba J}

Department of Pediatric Oncology, University Hospital Brno and Faculty of Medicine, Masaryk University Brno,

*Email ID: Melicharkova.Kristyna@fnbrno.cz

BACKGROUND: We are presenting the case report of 7 years old boy, who was diagnosed with stage III abdominal Burkitt lymphoma. Two months after completing standard chemotherapy, he was found to have an isolated radiological progression. We started with the retrieval therapy, but because of further early progression after the first cycle we switched on personalised experimental treatment. MATERIALS AND METHODS: The mutational analysis from the tumor proved germline mutation in PI3K delta subunit/ variant C830G at cDNA level and serine 312 cysteine on the protein level, which is outside the classical „Activated PI3K-delta syndrome“ (APDS) I or 2 variants. The mutational activation was confirmed by testing the patient's T- lymphocytes. The second biopsy on 9/20I5 showed a CD20 positive tumor, with high degree of proliferation and strong expression of PD-IL. The whole transcriptome analysis confirmed increased levels of PI3K and revealed additional HR23B (a good predictor of response to HDAC inhibitors). RESULTS: Based on these findings, we used idelalisib (a phosphoinositide 3-kinase inhibitor, which blocks PII0 0 , the delta isoform of the enzyme phosphoinositide 3-kinase). The single agent therapy led to normalization of the $\mathrm{S} 6$ (Ser235/236) phosphorylation in patient's peripheral T lymphocytes, but there was further disease progression. Additional combination of $\mathrm{CyVe/}$ idelalisib/obinutuzumab led to the disease stabilisation. The child received palliative 2 I Gy local radiation and based on the second biopsy findings, the nivolumab, a human IgG4 anti-PD-I monoclonal antibody, and valproic acid, class I selective HDAC inhibitor, were added. CONCLUSION: The patient has had partial response of the single residual abdominal tumor disease with no significant toxicity. He remains clinically well with Lansky score 100 and OS $>15$ months. His $3^{\text {rd }}$ EFS $/ 7$ months/ on the personalized therapy is already now his the longest EFS, compared to 6 months I Ist $^{2}$ FS on the standard BFM protocol and just I months after ibrutinib + ICE + anti CD20.

Is there a role for a novel maintenance therapy in pediatric patients with AML?

*Singh Randeep, Arora B, Nahar A, Prasad M, Gujral S, Subramanian PG,Amare-Kadam P, Banavali SD.

Tata Memorial Hospital, Mumbai.

PURPOSE: To study the clinical characteristics and outcome of children with acute myeloid leukemia (AML) and to determine the role of oral maintenance therapy (MT) in paediatric AML. PATIENTS AND METHODS: Any child from 0 - 18 years of age, diagnosed as de novo AML in last six years were included in the study. Any newly diagnosed AML who was treated with the institute specified protocol i.e. intensive induction (cytarabine and daunomycin- 
$3+7)$, three courses of high dose cytarabine $\left(18 \mathrm{gm} / \mathrm{m}^{2}\right)$ as consolidation and oral maintenance therapy (six months of oral 6-mercaptopurine and etoposide) were analyzed for treatment outcome. Patients with acute promyelocytic leukemia and Down syndrome were excluded. CNS prophylaxis consisted of administration of four doses of triple intrathecal, once, during each course of chemotherapy. RESULTS: One thirty nine children (0-I8 years), who were diagnosed as de novo AML, from the year 2004-09 and treated with the above specified protocol were studied. Out of 139 patients, 99 (7I.2\%) patients received standard induction. Of these 99 children who underwent standard induction, nine died due to chemo toxicity/severe infection $(9.0 \%)$ and one had primary refractory disease. We hereby present the characteristics and outcomes of 89 children who received induction, consolidation and maintenance therapy. At a median follow up of 5.7 years, the disease free survival (DFS) and overall survival (OS) was $64.0 \%$ and 69.0 $\%$ respectively. The relapse rate was $34.8 \%$. On univariate analysis, age, day 10 bone marrow blasts and cytogenetics were found to be insignificant predictors for overall survival. CONCLUSION: Our finding suggests that, the use of oral maintenance therapy gives favourably low incidence of relapse rates, thus regenerating interest to prospectively test this protocol with prolonged (twelve months) maintenance chemotherapy.

\section{METRONOMIC THERAPY IN SARCOMA}

Good Response to Metronomic Cyclophosphamide for Metastatic Epitheloid Hemangioendothelioma: Case Report

\section{Rohit Swami, Amit Joshi, Vanita Noronha, Vijay Patil, * Kumar Prabhash}

Tata Memorial Center,Mumbai, *Email ID: kprabhash I@gmail.com

INTRODUCTION: Epithelioid hemangioendothelioma (eHAE) is a rare tumor of vascular origin. Although it presents as low grade tumor ocassionaly, eHAE present as high grade and aggressive tumor. There is no standard chemotherapy treatment for eHAE at current but partial success with drugs such as Interferon, Paclitaxel, MAID, Doxurubicin, Pazopanib, Carboplatin,bevacizumab have been reported. We describe one patient treated with low dose oral cyclophosphamide, had good partial response and maintaining response since last II months on oral cyclophosphamide. CASE REPORT: A 55 year old Indian female, presented with multiple scalp nodules, left sided neck and preauricular nodule. Biopsy from neck nodule showed diffuse sheets, cords and focal papillary arrangements of polygonal shaped tumor cells. On IHC, tumor cells express CD3I while they were negative for CD34, Ck7, Ck20, p63, SI 00 and TTFI. Metastatic workup (PETCT) showed, no distant metastasis .Patient was treated with oral cyclophosphamide $50 \mathrm{mg}$ once a day and treatment response was monitored with 3 monthly whole body PETCT. Post 3 and 6 months of treatment PETCT showed stable disease. Post 9 month PETCT suggested partial response, with reduction of disease burden by $47 \%$ as per Resict criteria; with disease left at only one site in scalp. We added Celecoxib $100 \mathrm{mg} \mathrm{BD}$ to oral cyclophosphamide since last I month and only lesion persisting showed $25 \%$ reduction in size as per clinical measurements. DISCUSSION: Pro-Angiogenic factors are believed to promote the growth of eHAE. Metronomic chemotherapy has anti-angiogenic properties. Case reports had shown complete response to partial response in several eHAE cases. we achieved surprisingly good response with CMC.Because of clinical efficacy, good acceptance, low toxicity and low cost, CMC could be proposed as a new palliative therapeutic option.

Retrospective analysis of outcomes of patients with relapsed, refractory and metastatic sarcomas who have received metronomic chemotherapy

\section{Santosh Kumar, A Dongre, B Arora,T Vora, G Chinnaswamy, B Rekhi, S Laskar, S Kembhavi, A Puri, S D Banavali \\ Tata Memorial Hospital, Mumbai.}

INTRODUCTION: Relapsed and metastatic paediatric sarcomas have poor outcomes despite aggressive conventional chemotherapy, and local therapy. Metronomic chemotherapy has been used effectively with minimal toxicity especially in resource poor setting. We analyze the activity of metronomic in pediatric sarcomas. MATERIALS AND METHODS: This is retrospective, single institutional, observational study. We retrospectively reviewed data of South Asian Journal of Cancer • April-June 2016 • Volume 5 Issue 2 patients with relapsed, refractory or metastatic soft tissue sarcoma (STS) [ Ewing Sarcoma (ES);Rhabdomysarcoma (RMS) or other STS] who were treated with the metronomic protocol of oral Tamoxifen, Etoposide and Cyclophosphamide (TEC) during the period April 1998 to September 2013, at the Tata Memorial Hospital, Parel, Mumbai. Patients with ES and RMS were primarily treated on our Institutional protocols. The patients included in the analysis were those who had relapsed after the primary protocols and then treated with metronomic TEC protocol; or those with primary refractory or metastatic disease (RMS, ES) and received metronomic TEC therapy. RESULTS: 49 patients were enrolled. Among the 49 patients, 32 were diagnosed ES, I 3 RMS and 4 other STS. For the whole cohort response rates (RR) were $59 \%$ and clinical benefit rate (CBR) was $79 \%$. Patients in the study were grouped into the following subgroups. Systemic recurrent/ relapsed disease $(\mathrm{N}=24)$, metastatic disease at presentation $(\mathrm{N}=15)$ and local disease (refractory/recurrent) $(\mathrm{N}=10)$. None of the patients required blood or platelet support or admission for supportive care. The PFS for the above groups were 16.8 months, 12.5 months and 126.68 months respectively. This compares favorably with other historical cohorts in a similar setting. CONCLUSIONS: This study provides a preliminary evidence efficacy and tolerability of metronomic chemotherapy in poor risk ES and RMS. It also demonstrates that with this low cost low risk treatment few patients could go into long term remissions despite high disease burden.

\section{Patterns of Treatment and Outcomes in Fibromatosis at a Tertiary Cancer Center.}

\section{Gaurav Gupta, Ajay Puri,Ashish Gulia, Girish Chinnaswamy, Brijesh Arora, Suyash S. Kulkarni, Nitin Shetty, Siddharth Laskar, Nehal Khanna, N M Merchant, S L Juvekar, Bharat Rekhi, *S D Banavali}

Tata Memorial hospital, *Email ID: banavali_2000@yahoo.com

BACKGROUND: Metronomic chemotherapy has been used in patients of recurrent fibromatosis or patients who would require extensive, mutilating surgery with the aim to avoid such surgeries. The aim of this study was to assess the impact of systemic therapy on the outcome of patients with advanced / recurrent fibromatosis. METHOD: This was a retrospective analysis of Fibromatosis patients treated in TMH between January 2002 and December 20I2. The demographic details, presenting features, baseline investigations details, treatment details and outcomes were extracted from case record files and electronic medical records. Survival rates were estimated with the use of the Kaplan-Meier method. RESULTS: There were a total of 54 patients The median age at the time of presentation was 30 years. The number of pretreated and untreated patients at the time of presentation was equal in our study with 27 patients in each group. A total of 46 eligible patients received systemic therapy, 19 (4I.3\%) patients had primary tumors and $27(58.7 \%)$ had recurrent tumors. A total of $42 / 46$ (9l\%) patients were started MCT as first line systemic treatment at TMH, 4/46 (9\%) received other systemic therapies: only Imatinib $(n=I)$, Imatinib with Tamoxifen $(n=2)$ and Tamoxifen with celecoxib $(n=I)$. The median PFS with the use of MCT as the first line systemic therapy $(n=42)$ was 74.0 months ( $95 \% \mathrm{Cl}: 42.45$ to 105.54) with a PFS of $79.7 \% \pm 7.1 \%$ at 3 years. Amongst these, patients with primary disease had a median PFS of 74.0 months (95\% Cl:0.00 to 154.69) and patients with a recurrent tumor had a median PFS of 52.0 months $(95 \% \mathrm{Cl}$ : 44.2 I to 59.78). CONCLUSION: In patients with advanced desmoid tumors upfront use of systemic metronomic chemotherapy with surgery reserved for non-responding or patients progressing despite adequate therapy can be a reasonable approach. The regimen is easily and well tolerated; in most of the patients toxicities are easily manageable.

Update onmanagement of angiosarcoma using propranolol based metronomic chemotherapy

\section{Bhargava PG,AnuradhaChougule, Bharat Rekhi, Jaya Ghosh, Deepa S J Philip,Patil VM, *Banavali SD \\ Tata Memorial hospital, *Email ID : banavali_2000@yahoo.com}

BACKGROUND: Angiosarcomas are rare malignant tumours of vascular origin that represent a genuine therapeuticchallenge.Previous Pre-clinical and clinical evidence suggest that $\beta$-adrenergic receptor blockade may prove useful in the treatment ofangiosarcoma. Recently,we reported $100 \%$ response rate based onRECIST criteria, II months median PFS and 16 
months median OS in7 consecutive patients with advanced and/or metastatic/ recurrentangiosarcoma using an inexpensive combination treatment using propranolol based on pre-clinical data. MATERIALAND METHODS: All patients of angiosarcoma willing for metronomic chemotherapy were included in the study. Fifteen consecutive patients with advanced (3)/ metastatic (7)/recurrent (5) angiosarcoma were treated with a propranolol based metronomic chemotherapy, followed by propranolol-containing maintenance therapy. A combination using bi-daily propranolol $(40 \mathrm{mg})$ and weekly vinblastine $(6 \mathrm{mg} / \mathrm{m} 2)$ and methotrexate $(35 \mathrm{mg} / \mathrm{m} 2)$ was commonly used. Some patients were given propranolol with weekly paclitaxel or directly on oral metronomic chemotherapy. RESULTS: 3 patients had grade 3 or 4 toxicity( I thrombocytopenia, I diarrhea, I febrile neutropenia) and only I patient required discontinuation of treatment otherwise remaining tolerated well and resulted in 100\% response rate, including 6 complete response, 2 very good partial response, 6 partial responses and I stable disease, based on RECIST criteria. Median progression-free and overall survival was 10.6 months (range 5-24, 6 patients continue to show response till date) and 17.8 months (range 10-30, 9 patients have not reached OS) respectively. CONCLUSIONS: Our results provide a strong rationale for the combination of $\beta$-blockers and vinblastine-based metronomic chemotherapy for the treatment of advanced angiosarcoma. Furthermore, our study highlights the potential of drug repositioning in combination with metronomic chemotherapy in low- and middle-income country setting.

\section{METRONOMIC THERAPY IN GENITO-URINARY CANCER}

Weekly paclitaxel as metronomic therapy in metastatic urinary bladder cancer

\section{Vikas.T.Talreja, Amit Joshi, Vanita Noronha,Vijay Patil, * Kumar Prabhash}

Tata Memorial Hospital, *Email ID: kprabhashI@gmail.com

BACKGROUND:There is no standard second-line palliative chemotherapeutic option for urothelial carcinoma bladder post failure of gemcitabine- platinum -based first-line therapy. Hence this study was planned to explore the benefit of metronomic weekly paclitaxel in urothelial carcinoma bladder post gemcitabineplatinum failure. METHODS AND MATERIALS:This was a retrospective analysis done in Tata Memorial Hospital between January 2011 to December 2016. Demographic details, response, toxicities, compliance to treatment, and outcomes were recorded from electronic medical records and case file reports of consecutive patients who took treatment during this period. Primary end point was OS; secondary end points were PFS, ORR and toxicity. Statistical analysis was done by using SPSS version 16. RESULTS: There were 9 patients identified. The median age was 57 years (35-73). There were 7 males (78\%) and 2 females (22\%). The commonest site of metastasis was lung ( 6 patients). Median event free period post last line of chemotherapy was 4 months $(0-18)$ months. Weekly paclitaxel was administered as 2 nd line therapy in six patients and $3 r d$ line in three patients. Median numbers of cycles received were eight (8-32) All patients had stable disease at 2 months. Grade $3 / 4$ toxicities was noticed in I patient. Outcome data shall be presented at the conference. CONCLUSION:While the activity of single-agent taxanes in these heavily pretreated patients appears modest and needs to be explored further. I-5 department of medical oncology, Tata Memorial Hospital, Mumbai, Maharashtra , India.

\section{Oral Metronomic Chemotherapy in Castrate Resistant Prostate Cancer}

\section{Sharma Vibhor, Noronha Vanita, Joshi Amit, Patil Vijay M, *Prabhash Kumar}

Tata Memorial Hospital, *Email ID: kprabhash I@gmail.com

INTRODUCTION: In middle and low income countries, few treatment options are available for castrate resistant prostate cancer (CRPC) patients after they progress on docetaxel. OBJECTIVES:We report a case series of ten patients of metastatic CRPC treated with oral metronomic chemotherapy with focus upon progression free survival and treatment related toxicities. MATERIALSAND METHODS:This is a retrospective study of ten patients of metastatic CRPC treated at Department of Medical Oncology in Tata Memorial Centre, Mumbai. CRPC patients who had progressed on docetaxel or were unfit for docetaxel received oral metronomic chemotherapy. Data was obtained from electronic medical records of patients. RESULTS:This study includes 55 to 87 years old patients (median age 62.5years). Five patients each had ECOG performance status I and 2 respectively. Gleason's score ranged from 7 to 10 (median score 8). Bone and lymph nodal metastases were present in nine and four patients respectively. Time from diagnosis to progression to CRPC state ranged from 7 to 104 months (median 38months). Before receiving oral metronomic chemotherapy, six patients had received docetaxel. Four patients were deemed unfit for docetaxel due to poor performance status, inaffordability, patient choice or advanced age. For nine patients, the metronomic chemotherapy regime included tablet cyclophosphamide $50 \mathrm{mg}$ od for $2 \mathrm{I}$ days on $/ 7$ days off and tablet dexamethasone $0.5 \mathrm{mg}$ od continuously. One patient received only tablet dexamethasone $0.5 \mathrm{mg}$ od. There were no grade 3/4 toxicities. Two patients had hyperglycemia and one each had transaminitis and hypertension respectively. Progression free survival ranged from 3 to 12 months (median 4 months; mean 5.4months). Three patients were responding till their last evaluation. CONCLUSIONS: Oral metronomic chemotherapy consisting of cyclophosphamide and dexamethasone is well tolerated and produces durable response occasionally. This regimen may be used in resource constrained countries. Larger studies involving more patients need to be carried out.

KEYWORDS: Metronomic, Chemotherapy, CRPC

\section{BRIEF REPORTS: METRONOMIC THERAPY IN CNS CANCER}

A one year four-drug metronomic regimen as alternative treatment for recurrences from high- risk neuroblastoma

\section{*Frank Berthold', Jaroslav Sterba², Inna Proleskovskaya ${ }^{3}$, Pavel Mazanek ${ }^{2}$, Margarita Belogurova ${ }^{4}$ Marc Hömberg', Thorsten Simon', Barbara Hero', Ruth Volland'}

Departments of Pediatric Oncology and Hematology of the Universities of 'Cologne (Germany), ${ }^{2}$ Brno (Czech Republic), ${ }^{3}$ Minsk (Belarus),

${ }^{4}$ St. Petersburg (Russia), *Email ID: frank .berthold@uk-koeln.de

BACKGROUND: Survival rates and times are discouraging for patients with recurrences from high risk neuroblastoma. The median times from first to second progression was 4 months and to death 9 months only in a large series $(n=521)$. Second line treatment consisted of multiagent cyclic chemotherapy with and without myeloablation. One third $(35 \%)$ of the patients chose palliative support. Here we report the first results with pilot patients of a new metronomic concept. PATIENTS AND METHODS: This pilot study included 20 patients with recurrent high-risk neuroblastoma. The treatment was administered on an outpatient basis and consisted of Celecoxib (antineuroblastic, effect on microenvironment) $2 \times 200 \mathrm{mg} / \mathrm{m}^{2} \times \mathrm{d} \mathrm{dl}-365$ oral, Cyclophosphamide (anti-neuroblastic, anti-angiogenetic) I $\times 25 \mathrm{mg} / \mathrm{m}^{2} \times \mathrm{d}$ dl -365 oral, Etoposide (anti-neuroblastic, anti-angiogenetic) I $\times 25 \mathrm{mg} / \mathrm{m}^{2} \times \mathrm{d}$ $\mathrm{dI}-2 \mathrm{I}$ for 4 cycles oral, andVinblastin (anti-neuroblastic, anti-angiogenetic) Ix $3 \mathrm{mg} / \mathrm{m}^{2} x$ d every 14 days i.v.. The treatment was administered until event and if no event occurred up to 24 months. RESULTS: I patient had I recurrence, 19 patients 2-4 recurrences before start of metronomic therapy. 5 children had one, II two sites and I patient three recurrence sites (primary tumor I2, osteomedullary 12, CNS 3, liver I, lymph node I). The toxicity was minimal except grade 2-3 for thrombocytopenia, leukopenia, anemia. 3 patients in CR after preceding therapy were excluded from survival analysis comparing 17 metronomically $(\mathrm{M})$ with 307 conventionally $(\mathrm{C})$ treated patients. The I year secondary EFS was $64 \pm 12 \%(M)$ vs. $36 \pm 3 \%(C)(p=0.271)$, the I year secondary OS $79 \pm 1$ I vs. $57 \pm 3 \%(p=0.052)$. The number of recurrence sites $(I />I)$ and time from diagnosis to first recurrence ( $</>\mid 8$ months) were equally distributed over the different groups. CONCLUSION: The metronomic approach in recurrent high-risk neuroblastoma is at least not inferior to the maximum tolerated dose concept, but minimal toxic. An amended 5 drug prospective trial including propranolol is underway.

\section{METRONOMIC THERAPY}

Germinal mutation of PDGFR alpha in patient with tuberous sclerosis complex

\section{*Melichárková K, Zapletalová D, Štěrba J}

Department of Pediatric Oncology, University Hospital Brno and Faculty of Medicine, Masaryk University Brno,

*Email ID: Melicharkova.Kristyna@fnbrno.cz 
BACKGROUND:We are presenting the case report of 9 years old boy with tuberous sclerosis complex. Within that he has angiomyolipomas of kidneys, bilateral hamartomas of retina, pharmacoresistant epilepsy, hypothyreosis. I I/20I 4 he was diagnosed with malignant PEComa in the abdominal cavity with the residual disease after the surgery. MATERIALSAND METHODS: The mutational analysis from the tumor tissue and then also from the peripheral blood cells proved germinal mutation in PDGFRa - substitution in exon 10. Looking into the literature it remains unclear if this mutation leads to an activation of the protein, but the relationship to the PEComa in this case is suspicious. The analysis of the profile of phosphorylated proteins in the tumor cells revealed highly activated EGFR, InsR, IGF-IR and PDGFR $\beta$. RESULTS: Due to these results we started with personalized treatment encompassing everolimus/sunitinib/metformin oraly: The patient is now without measurable disease according to the ultrasound with EFS/OS 16 months. CONCLUSION: The coincidence of tuberous sclerosis complex with another germinal mutation is rare.This case report shows the possibility to use combination of different targeted therapies, which can help to stabilize/ cure malignant tumors in such patients.

\section{Insilico study of tyrosine kinase active site and angiogenic inhibitors}

\section{*Surya Pratap Singh, Krishna Misra \\ Indian Institute of Information Technology, Allahabad, U.P, \\ *Email ID:vininnair@gmail.com}

The tyrosine kinases are the key targets for the reason that; they play a key role in the modulation of growth factor signaling. The small inhibitor molecules of tyrosine kinase contend with the ATP binding site of the catalytic domain of various oncogenic tyrosine kinases. These are small orally active molecules that are having a favorable safety profile and can be easily mixed with the other forms of chemotherapy or radiation therapy, many tyrosine kinase inhibitors have been found to have effective antitumor activity and have been approved and some these are in clinical trials. The tyrosine kinases are the key intermediators for the signaling cascade, which determining the important roles in different biological processes like apoptosis, differentiation, metabolism and growth in response to external and internal stimuli. The key role of tyrosine kinases in the pathophysiology of cancer are recently going on progresses. Although their specific activity is tightly modulated in normal cells, they can adopt transforming functions due to autocrine paracrine stimulation, overexpression, mutation and leading to malignancy. The essential oncogenic activation in cancer cells can be choked up by selective tyrosine kinase inhibitors. The ways of oncogenic activation and the different kind of comings for tyrosine kinase inhibition are like antisense, heat shock proteins, monoclonal antibodies, immunoconjugates, small molecule inhibitors and peptide drugs are brushed up in light of the significant molecules. The angiogenesis is a key event for cancer cell proliferation and growth, tyrosine kinase inhibitors are the effective targets for anti-angiogenesis that can be well applied as a new mode of cancer therapy. In the process of angiogenesis the new blood vessels are formed from pre-existing ones through the proliferation of vascular endothelial cells. It is an important physiological process in the growth of cancer cells still it play an important role in the progression of human diseases such as atherosclerosis, diabetic-retinopathy and cancer.The events of the most cogent angiogenic inhibitors that counteract the effects of vascular endothelial growth factor and basic fibroblast growth factor have recently been known. Angiogenic inhibitors are intermediated through cell surface receptors that are having integral protein tyrosine kinase activity. However, the mechanism by which these different angiogenesis inhibitors put to use their common effects remains largely unidentified. A variety of well characterized angiogenesis inhibitors (including angiostatin, thalidomide, 2-methoxy estradiol, transforming growth factor and fumagillin) effectively blocked vascular endothelial growth factor. PDI73074 (PDB code 2FGI) is a specific inhibitor of mitogen-activated protein kinase and it is a known angiogenesis inhibitor, it also blocked the observed vascular endothelial growth factor.

Metronomic chemotherapy: (Re)assessing its anti-tumor mechanisms

\section{Robert S. Kerbel}

Sunnybrook Research Institute, University of Toronto,

Email ID: robert.kerbel@sri.utoronto.ca
The original mechanism hypothesized or shown to explain the mechanism of chronically administering chemotherapy in a continuous or frequent low-dose ('metronomic') fashion with no extended breaks was via antiangiogenesis. There are at least four ways this can occur: I) by inducing apoptosis in 'activated' endothelial cells of the tumor's angiogenic neovasculature; 2 ) inhibiting the mobilization and tumor homing of proangiogenic bone marrow-derived cells (BMDCs), e.g. endothelial progenitor cells (EPCs), and by suppression of the HIF-I $\alpha$ transcription factor. However, from the start (in 2000), another possible mechanism is stimulation of the immune system by suppression of T regulatory cells when using certain drugs such as cyclophosphamide; 3) a third mechanism is by direct inhibitory effects on the proliferation and survival of tumor cells, and a fourth, is through an anti-vascular effect that is independent of inhibiting tumor angiogenesis. Experimental findings will be discussed which will focus on the third and fourth mechanisms, utilizing various models of metastatic disease in mice, and which involve results that are not necessarily consistent with an antiangiogenic mechanism, but rather direct tumor cell targeting effect, and possibly an effect on co-opted ('hijacked') established vessels in tumors growing in sites such as the lungs or liver.

The above brief summaries include a total of thirty studies containing data on 1390 patients with various malignancies (544 with head neck cancer, 153 with ovarian cancer, 260 with breast cancer, 118 with lung cancer, 147 with leukemia and lymphoma, 129 with sarcoma, 19 with genitourinary cancer, and 20 with central nervous system cancers).

The 12 abstracts in head neck cancer document the combined experience in a total of 544 patients. If we exclude the 15 patients treated with weekly paclitaxel and carboplatin, it still is a collective data on 516 cases. The most common metronomic therapy combination used was methotrexate and celecoxib, drugs that are easily available and inexpensive. ${ }^{[1]}$ One study of 13 patients included the addition of erlotinib as the third drug. We would like to comment on two specific studies. Abstract No. 3 by Agarwala et al. involving sixty patients clearly shows that conventional standard systemic therapy (paclitaxel plus cetuximab) is significantly better than oral metronomic therapy (OMT). These data indicate that OMT should not be used if the patient is able to receive standard of care. ${ }^{[2]}$ Abstract No. 8 by Chandrakanth et al. compared quality of life (QoL) in patients receiving OMT versus weekly cisplatin. These results clearly show that QoL is same in both the arms. ${ }^{[3]}$ What is most interesting is the data of 110 patients reported by Patil et al. This impressive prospective randomized study showed that both overall survival (OS) and progression-free survival (PFS) was better with OMT as compared to weekly paclitaxel, with a $P=0.02$. Moreover, this was achieved with fewer adverse events as well. Such comparison has also been made between OMT and weekly cisplatin. ${ }^{[4,5]}$ This is the most impressive data that we have seen in favor of OMT so far.

The three abstracts on ovarian cancer used etoposide, cyclophosphamide, tamoxifen, erlotinib, and/or celecoxib as agents for OMT. These data need cautious interpretation. Clearly in the neoadjuvant setting, the standard therapy of paclitaxel and platinum gave a response rate of almost 100\% (34/35 cases) and better median PFS as reported by Pandey et al. In heavily pretreated patients, the value of OMT is in providing an OS of 7 months as shown by Kalaichelvi et al. Gupta et al. have shown that use of etoposide, cyclophosphamide, and tamoxifen is not without significant adverse events, with more than half the patients requiring 
dose reduction. ${ }^{[6]}$ The value of this strategy, however, was in obtaining an overall response rate (ORR) of $76 \%$.

The data on 260 women with breast cancer from rural India by Khatwani et al. show that there is a lot of scope for improvement. If an ORR of $25 \%$ can be obtained in metastatic triple negative breast cancer for the subset who had failed previous therapy with standard therapy, use of oral cyclophosphamide and methotrexate could be a valuable option, especially in the low- and medium-resource setting. ${ }^{[7]}$

In fact, the most interesting adjuvant trials are the ABCDE trial (NCT00925652) where two of the four arms receive metronomic cyclophosphamide with bevacizumab (plus diet intervention \pm exercise), the SYSUCC-001 trial (NCT01112826) in TNBC patients where the intervention group receive 1 year of metronomic capecitabine, and the IBCSG 22-00 trial (NCT00022516) using cyclophosphamide-methotrexate maintenance after standard adjuvant therapy in hormone receptor negative patients. ${ }^{[8,9]}$

The data on 139 patients with acute myeloid leukemia by Singh et al. indicate that there could be potential benefit from maintenance therapy, especially if it can contribute to achieving an OS of $69 \%$ at 5.7 years. ${ }^{[10,11]}$

Among patients with sarcoma, the drugs used as OMT included tamoxifen, etoposide, cyclophosphamide, propranolol, vinblastine, and methotrexate. An ORR of $59 \%$ in the study by Kumar et al. in the setting of refractory, relapsed and metastatic sarcoma shows the potential of this approach.

More than a decade ago, Kerbel and Kamen independently published about the antiangiogenic effects of low-dose chemotherapy a strategy that was subsequently made popular as metronomic chemotherapy. ${ }^{[12,13]}$

Kerbel and Kamen also show that OMT is likely to have more than the usually recognized antiangiogenic effect. This includes, but is not limited to, targeting endothelial cells of the tumor neovasculature; inhibiting mobilization or directly targeting proangiogenic bone marrow-derived cells; suppressing hypoxia-inducible factor1-apha; direct action against cancer stem cells and indirect action via stimulating the immune system. ${ }^{[13]}$

Medline search "metronomic chemotherapy India" yielded 31 references, 17 of which are involving the Department of Medical Oncology, Tata Memorial Hospital, Mumbai (http://www.nlm.nih.gov/). Hence, it is not surprising that the international community awarded the first Barton Kamen-MGHI Prize to Prof. Shripad Banavali in 2014. This prize was given by the wife of the late Dr. Barton Kamen, a pediatric oncologist, who pioneered low-dose chemotherapy and was an untiring advocate of metronomic chemotherapy. ${ }^{[9]}$

While it has been showcased as a therapeutic strategy for patients living in low- and middle-income countries, it's obvious benefit of repurposing old drugs at miniscule cost and significantly lower toxicity, makes it applicable globally even among the wealthy nations. ${ }^{[14,15]}$
By bringing out an issue of the Indian Journal of Cancer on metronomic therapy in 2013, India led the initiative instead of simply copy-pasting so-called standard-of-care from the western world. ${ }^{[8]}$ Moreover, by hosting the international metronomic conference in Mumbai, India, this year (which is the next logical step), India is cementing its leadership role in this field.

It is now time for various thought leaders, think tanks, governments, pharmaceuticals companies, and international NGOs to join hands to initiate a global strategy and program to systematically study, document, define, and embed the exact place of metronomic chemotherapy in the armamentarium against cancer. Incorporating strategies to design metronomic chemotherapy trial, with biomarkers and reliable surrogate markers of clinical benefit, will enable oncologists to optimize its value in the current era of personalized medicine. ${ }^{[16-18]}$

P. M. Parikh, S. S. Hingmire', C. D. Deshmukh' Department of Precision Oncology, Asian Cancer Institute, Sion East, Mumbai, 'Department of Oncology, Deenanath Mangeshkar Hospital, Erandwane, Pune, Maharashtra, India Correspondence to: Dr. Parikh M. Parikh, E-mail:purvishI@gmail.com

\section{References}

1. Patil V, Noronha V, Krishna V, Joshi A, Prabhash K. Oral metronomic chemotherapy in recurrent, metastatic and locally advanced head and neck cancers. Clin Oncol (R Coll Radiol) 2013;25:388.

2. Patil V, Karpe A, Noronha V, Joshi A, Muddu V, Bhattacharjee A, et al. Efficacy of second-line erlotinib in patients postprogression of first-line chemotherapy in head and neck cancers. Indian J Cancer 2015;52:629-31.

3. Noronha V, Joshi A, Marfatia S, Patil V, Juvekar S, Arya S, et al. Health-related quality of life in patients with metastatic, relapsed, or inoperable squamous cell carcinoma of the head and neck in India. Support Care Cancer 2016;24:1595-602.

4. Patil VM, Noronha V, Joshi A, Muddu VK, Dhumal S, Bhosale B, et al. A prospective randomized phase II study comparing metronomic chemotherapy with chemotherapy (single agent cisplatin), in patients with metastatic, relapsed or inoperable squamous cell carcinoma of head and neck. Oral Oncol 2015;51:279-86.

5. Pai PS, Vaidya AD, Prabhash K, Banavali SD. Oral metronomic scheduling of anticancer therapy-based treatment compared to existing standard of care in locally advanced oral squamous cell cancers: A matched-pair analysis. Indian J Cancer 2013;50:135-41.

6. Majumder D, Mukherjee A. Mathematical modelling of toxicity-related trade-offs in metronomic chemotherapy. IET Syst Biol 2007; 1:298-305.

7. Noronha V, Tsomo U, Jamshed A, Hai M, Wattegama S, Baral R, et al. A fresh look at oncology facts on south central Asia and SAARC countries. South Asian J Cancer 2012;1:1-4.

8. André N, Pasquier E. Metronomics in low and middle income countries: India showing the way! Indian J Cancer 2013;50:112-4.

9. Bouche G, André N, Banavali S, Berthold F, Berruti A, Bocci G, et al. Lessons from the fourth metronomic and anti-angiogenic therapy meeting, 24-25 June 2014, Milan. Ecancermedicalscience 2014;8:463.

10. Tandon N, Banavali S, Menon H, Gujral S, Kadam PA, Bakshi A. Is there a role for metronomic induction (and maintenance) therapy in elderly patients with acute myeloid leukemia? A literature review. Indian J Cancer 2013;50: 154-8.

11. Malik PS, Raina V, André N. Metronomics as maintenance treatment in oncology: Time for chemo-switch. Front Oncol 2014;4:76.

12. Browder T, Butterfield CE, Kräling BM, Shi B, Marshall B, O'Reilly MS, et al. Antiangiogenic scheduling of chemotherapy improves efficacy against experimental drug-resistant cancer. Cancer Res 2000;60: 1878-86. 
13. Kerbel RS, Kamen BA. The anti-angiogenic basis of metronomic chemotherapy. Nat Rev Cancer 2004;4:423-36.

14. Noronha V, Krishna MV, Patil V, Joshi A, Banavali SD, Prabhash K. Metronomic therapy: Chemotherapy revisited. Indian J Cancer 2013;50:142-8.

15. Bahl A, Bakhshi S. Metronomic chemotherapy in progressive pediatric malignancies: Old drugs in new package. Indian J Pediatr 2012;79:1617-22.

16. Agrawal A, Purandare N, Shah S, Puranik A, Banavali S, Rangarajan V. Response assessment in metronomic chemotherapy: RECIST or PERCIST? Indian J Nucl Med 2014;29:74-80.

17. Sondhi V, Arun Kurkure P, Jalali R, Rangarajan V, Sridhar E, Medhi S, et al. Complete remission and long-term survival in a child with relapsed medulloblastoma with extensive osteosclerotic bony metastasis with a novel metronomic chemobiological approach. J Pediatr Hematol Oncol 2012;34:e 195-8.

18. Parikh PM, Prabhash K, Bhattacharyya GS, Sirohi B, Rajappa S, Verma A, et al. Ignore molecular oncology at your peril. Indian J Cancer 2014;51:150-3.
This is an open access article distributed under the terms of the Creative Commons Attribution-NonCommercial-ShareAlike 3.0 License, which allows others to remix, tweak, and build upon the work non-commercially, as long as the author is credited and the new creations are licensed under the identical terms.

\begin{tabular}{|l|l|}
\hline \multicolumn{2}{|c|}{ Access this article online } \\
\hline Quick Response Code: & Website: \\
\hline & www.sajc.org \\
\cline { 2 - 2 } & \\
\hline
\end{tabular}

How to cite this article: Parikh PM, Hingmire SS, Deshmukh CD. Selected current data on metronomic therapy (and its promise) from India. South Asian J Cancer 2016;5:37-47.

The Molecular Oncology Society announces the $2^{\text {nd }}$ MOSCON, the society's annual conference. It will be held on $28^{\text {th }}$ and $29^{\text {th }}$ January 2017 at Ahmedabad. For further details please contact Dr. Bhavesh Parekh, Org Secretary (bhaveshdm1@hotmail.com) or Dr. Purvish Parikh (purvish1@gmail.com)

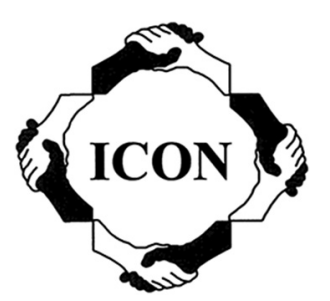

The Indian Cooperative Oncology Network is proud to announce its $35^{\text {th }}$ ICON Conference being organized from $9^{\text {th }}$ to $11^{\text {th }}$ September 2016 at Mumbai. For further details please contact

Dr. Kumar Prabhash (kprabhash1@gmail.com) or

Dr. Purvish M. Parikh (purvish1@gmail.com)

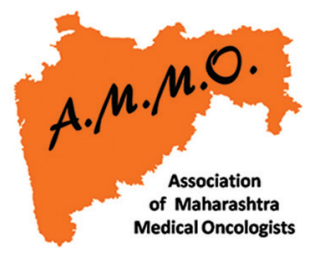

The Association of Maharashtra Medical Oncologists brong to you their $2^{\text {nd }}$ AMMO Conference in Nagpur in the month of August 2016. For further details please contact Dr. Padmaj Kulkarni (padmaj.kulkarni@gmail.com) or Dr. AA Ranade (draaranade@yahoo.com) 